\title{
IMPACT OF ELECTRONIC HEALTH RECORDS ON PATIENT OUTCOMES
}

\author{
Lilian Ndifon limechi@hotmail.com \\ Jude E. Edwards, Washington Adventist University, drjudeedwards@gmail.com \\ Leila Halawi, Embry Riddle Aeronautical University, halawil@erau.edu
}

\begin{abstract}
With the passing of the HITECH Act, EHRs have come into prominence and sharper focus, due to efforts by the government to push for a national adoption of EHRs into our healthcare system. This push for a national adoption of EHRs is based on the premise that it will help improve the quality delivery of health care services and reduce costs. However, this push for a "national adoption" has experienced mixed results. This study was designed to assess the impact of EHRs post-2009, the year of the HITECH Act, to review some of the key contributing factors to this mixed results. The findings of the study show that implementing EHRs has positively impacted the US health care system in a variety of ways. Thus, while we may conclude that the implementation EHRs have definitely improve the quality of healthcare in the US, and show great promise for the future of health care in this nation, there is still much work to be done to ensure that their full potential is realized. These findings will contribute towards the ongoing effort to expand the implementation of HER by making them secure and easy to use
\end{abstract}

Keywords: Electronic Health Record, Health Information, HITECH Act, Healthcare System, Information Exchange, Medical Errors, Health Information Technology

\section{INTRODUCTION}

According to Versel (2013), "Electronic health records (EHRs) have been around in the US in one form or another since the 1960s, but the notion of patient records being stored on computers is only beginning to seep into the public's consciousness. While pretty much every other industry computerized years ago, the vast majority of Americans' medical records remain on paper".

For many years, the Institute of Medicine had been urging the medical community in the United States to adopt electronic health record arguing that it would improve the quality of healthcare by making it safer and more efficient. In 2004, President G.W. Bush undoubtedly influenced by the institute's call, expressed the wish that most Americans should have electronic medical records within a 10-year period. To this end, in April 2004 he signed executive order number 13335 (69FR24059) establishing the Office of National Coordinator (ONC) for Health Information Technology in the Department of Health and Human Services to oversee the implementation of a national EHR program. The ONC quickly devised a strategic plan that aimed to provide "consumer-centered and information-rich Health Care". The plan was based on the following three assumptions:

- Ample research evidence that Health Care IT saves lives and money

- Benefits of Health Care IT far exceed its costs, hence the need for the federal government to take the lead and provide incentives for its adoption.

- Demand for a national Health Care IT is overwhelming.

The idea was to develop a web-based National Health Information network while protecting privacy and personal health information of consumers. In July, 2005 a public-private partnership known as the American Health Information Community was formed. The Community then created an Electronic Health Records workgroup. Based on the recommendations of the workgroups the Department of Health and Human Services then awarded contracts for Standards Harmonization, HIT Compliance Certification, development of a prototype architecture, Security and 
Privacy, and an Adoption Study. In April 2008, the National Alliance for Health Information Technology released operational definitions that would be employed in developing and deploying the electronic health record networks. At its inception, the Obama administration made health care reform the centerpiece of its health policy focusing on affordable care, universal coverage, improving performance and efficiency, reducing costs, reducing waste, and promoting the use of Electronic Health Records for information exchange between care providers. In 2009, the President signed into law the American Recovery and Reinvestment Act (ARRA) also known as the Economic Stimulus Act. Included in ARRA was the Health Information Technology for Economic and Clinical Health, or the HITECH Act. ARRA caused the creation of two federal committees - the Health Information Technology Policy Committee and the Information Technology Standards Committee to complement the National Committee on Vital Statistics that was already in existence. ARRA also allowed for the allocation of $\$ 25.8$ billion for investment in health information technology and the provision of incentive payments to promote its adoption by healthcare providers nationwide.

Thus, with Electronic Health Record becoming more widespread, it is worthwhile to examine its effects on the nation's healthcare management to date. This paper focuses specifically on the adoption of EHRs in healthcare management in the United States since 2009 to date and the impact on patient outcomes. The aim is to shed some light on what is working or not working with a view to highlighting a possible way forward. In addition, the US healthcare system has become tremendously complex and there is no doubt that such a very complex system can only be managed effectively with a twenty-first century management technology such as the EHR. This is particularly important in view of the changing way of life of today's increasingly mobile and digitally connected citizenry.

After this section, we present a review of the literature on EHRs and attempt to summarize arguments in favor of its adoption and the benefits it promises to deliver to our health care system. We also briefly discuss government efforts to encourage the adoption of EHRs as well as the paucity of empirical data to support the claims being made in support of it. The findings of the study - the impact of EHRs on clinical outcomes, patient perception and satisfaction, hospital readmissions, patient monitoring and compliance, and patient safety are discussed in the next section. Finally, the conclusion and suggestions for future research are presented.

\section{LITERATURE REVIEW}

\section{Background - Electronic Health Records (EHRs)}

Electronic Health Records (EHRs) are sometimes called Electronic Patient Records, Computerized Patient Records, or Electronic Medical Records (EMRs). In the present study they will only be referred to as Electronic Health Records. An EHR is a digital or computerized record of a patient's medical or health history that is kept in a doctor's office or any medical establishment and can be shared between medical agencies, government agencies, doctors etc. Computerized, simply means generated by computer and stored in a computer

\section{The Argument for Electronic Health Records}

For many years, the Institute of Medicine urged the medical community in the United States to adopt electronic medical records arguing that it would improve the quality of the nation's healthcare. In 2001, the institute issued a report titled, "Crossing the Quality Chasm", in which it detailed the reasons for the gaps in quality and fervently advocated the expansion of healthcare IT infrastructure as a way of addressing the problem of quality. The report stated, "The U.S. health care delivery system does not provide consistent, high-quality medical care to all people. Americans should be able to count on receiving care that meets their needs and is based on the best scientific knowledge--yet there is strong evidence that this frequently is not the case. Health care harms patients too frequently and routinely fails to deliver its potential benefits. Indeed, between the healthcare that we now have and the healthcare that we could have lies not just a gap, but a chasm" (IOM, 2001). The report then goes on to identify the factors responsible for the low quality of healthcare such as "growing complexity of health care, which today is characterized by more to know, more to do, more to manage, more to watch, and more people involved than ever before" (IOM, 2001). 
The authors of the report argued that given these challenges and other "challenges such as the increase in life span of Americans accompanied by an increasing incidence of chronic conditions such as heart disease, diabetes, and asthma, the poor organization, and overwhelming complexity of the current system, given the lack of coordination whereby "healthcare organizations, hospitals, and physician groups typically operate as separate "silos," acting without the benefit of complete information about the patient's condition, medical history, services provided in other settings, or medications provided by other clinicians, and given its cumbersomeness, and waste the nation's healthcare delivery system has fallen far short in its ability to translate knowledge into practice and to apply new technology safely and appropriately"(IOM, 2001).

Therefore, a plan of action was adopted which was aimed were to make healthcare safe, effective, patient-centered, timely, efficient, and equitable. To achieve these aims, it was suggested that some criteria that should be considered when redesigning a health care system - "care must be based on continuous healing relationships, customized according to patient needs and values, patient must be the source of control, shared knowledge and free flow of information, safety as a system property, transparency, anticipated needs, continuous decrease of waste, and cooperation among clinicians" (IOM, 2001). In our view, all of the stated aims revolve or are connected to accurate, timely, and easy availability of information which can only be possible through the use of information technology.

It was therefore no surprising that one of the areas identified by the report as urgently in need of change, in order to achieve the aims stated above, was the use of information technology. The argument was made that "Information technology, including the Internet, holds enormous potential for transforming the nation's healthcare delivery system. Central in many information technology applications is the automation of patient-specific clinical information. Such information typically is dispersed in a collection of paper records, which often are poorly organized, illegible, and not easy to retrieve, making it nearly impossible to manage various illnesses, especially chronic conditions, that require frequent monitoring and ongoing patient support. Many patients also could have their needs met more quickly and at a lower cost if they could communicate with health professionals through email. In addition, the use of automated systems for ordering medications can reduce errors in prescribing and dosing drugs, and computerized reminders can help both patients and clinicians identify needed services" (IOM, 2001). Many other researchers - Bates \& Gawande, 2003; Alvarez, 2005; Wolfe, 2008; Gonzalez, 2013) just to mention a few have made similar arguments in favor of EHRs. These reports without a doubt must have informed the decisions taken by Presidents G.W. Bush and Obama in their efforts to improve the health care system of the United States.

\section{The Promise of Electronic Health Records}

According to Gonzalez (2013), the benefits of a national EHR system include "care continuum, cost reduction, medications management, long-term perspective and portability, emergency episode management and patient empowerment."

However, everyone knows that it is one thing for a system to promise a lot of potential and another thing entirely for it to deliver on that promise. That is the crux of the present study - to see if EHRs are living up to the hype in terms of improving the quality of healthcare.

\section{Government Incentives for EHR adoption}

As the saying goes, change is not easy. Unfortunately, electronic health records are no exception to this fact of life. The reasons for this include, costs of adoption of EHR, reluctance on the part of some providers to transition, lack of training on the part of doctors, nurses and other medical staff in the use of EHRs, privacy concerns, and in some cases a sheer refusal of some people to accept change or to see the benefits of EHR.

Cognizant of these realities, the federal government put in place Medicare and Medicaid Incentive Programs to make payments to eligible professionals and eligible hospitals as a way of encouraging them to adopt, implement, upgrade or demonstrate meaningful use of certified EHR technology. Thus, ARRA also allowed for the allocation of $\$ 25.8$ billion for investment in health information technology and the provision of incentive payments to promote its adoption by healthcare providers nationwide. With this, professionals deemed eligible can receive up to $\$ 44,000$ from the Medicare EHR Incentive Program and up to $\$ 63,750$ from the Medicaid EHR Incentive Program. The 
Medicare EHR incentive program began in 2011 and will continue through 2016. Professionals can participate in the program for up to 5 continuous years. Maximum EHR incentive payments accrue to those professionals that joined the program by 2012 and who continue to demonstrate meaningful use of EHR for each year of participation. If a Medicare eligible professional predominantly provides care or services in one of the areas designated as Health Professional Shortage Area they will be eligible for a $10 \%$ increase in their annual EHR incentive payments. The Medicaid EHR Incentive Program is offered and administered by states and territories that so choose. State participation is voluntary. Payments of EHR incentives through this program will continue through 2021. Eligible professionals who decide to participate in the program must be enrolled by 2016. They can participate for up to 6 years. Professionals that qualify for both Medicaid and Medicare EHR incentive payments must choose one or the other, not both, but Medicaid eligible professionals receive a payment adjustment if they also treat Medicare patients.

In reviewing the literature on the impact of EHRs on quality of healthcare in general and patient outcomes in particular, the first thing that strikes one is the abundance of anecdotal evidence and the appalling dearth of empirical data. This situation may have led Reed, Huang, Brand et al (2013) to remark that, "although the use of EHRs has the potential to improve patient health outcomes, the existence of evidence has been limited and inconsistent. The reason for scarcity of evidence was not specifically addressed by any researcher. However, comments made by a variety of other researchers revealed some interesting facts that collectively may shed some light on the probable reasons for this situation. For example, Poissant, Pereira \& Tamblyn (2005) found that a computerized physician order entry (CPOE) was inefficient since it significantly increased physician workload by increasing their work time per working shift. Strangely enough the same study found that use of CPOEs saved nurses about $25 \%$ of the overall time spent documenting during a shift. According to them, there were three main reasons for this difference: the type of terminal used (nurses used bedside and central stations while doctors used only central stations), user characteristics and training, and the technical characteristics supporting an EHR system such as automation. Hopkins (2013) reports on a study that finds that the degree to which EHRs are implemented could be a problem in assessing their impact. The report claims that some doctors make only minimal use of EHRs in their practice hence he argues in which case, the widespread adoption of EHRs may not change how some doctors diagnose and treat patients after all.

In addition to the above reasons, it appears that the problem of defining quality may have also been a contributing factor to this dearth in evidence. Thus, the American Heart Association/American College of Cardiology First Scientific Forum, on Assessment of Healthcare Quality in Cardiovascular Disease of 2000, came to the conclusion that measuring and monitoring health care is very important, but that quantifying healthcare quality is a very complex and challenging task. The forum defined a health outcome as, "the consequences of treatment which can represent markers of disease progression (mortality, readmission, etc), health status (symptoms, functioning, and quality of life), and/or cost"(IOM, 2000). Parsons, McCullough, Wang et al (2012) in a study supported by the National Agency for Healthcare Research and Quality identified a need for more studies to determine which quality measures are best suited for automated accounting. They also stressed the importance of additional training for care providers to increase their accuracy of documenting health events. They concluded that "EHRs offer new potential for performance measurement .....however, EHR-derived quality measurement has limitations due to several factors, most notably variations in EHR content, structure and data format, as well as local data and extraction procedures" (Parsons, McCullough, Wang et al ,2012). Similar views were expressed by Galanter, Hier, Jao et al (2010) and Chan, Fowles, \& Weiner (2010).

\section{METHODOLOGY}

This qualitative exploratory study is designed to examine the impact of Electronic Health Records on Patient Outcomes. While some quantitative results are presented, (specifically, figures $1 \& 2$ and tables $1 \& 2$ ), those results were not determined using "primary" data collection and analytical methods, but from a variety of "secondary" sources. A number of key indicators were selected and reviewed to gauge and measure this impact. These key indicators were: (a) The Promise of Electronic Records, (b) Government Incentives for HER Adoption, (c) the Impact on Clinical Outcomes; (d) the Impact on Patient Perception and Satisfaction; (e) the Impact on Hospital Readiness; (f) the Impact on Patient Monitoring and Compliance; (g) and the Impact on Patient Safety. The results 
of this review of applicable sources was then used to access the overall impact of EHRs on patient outcomes. A key area that this research did not address is the matter of EHR security, as cyber-security, or the lack thereof, might also be a key contributor to the impact of EHR on patient outcomes. This is addressed in the section that addresses future research.

To assess the impact of a management product is to look at its effect. Effects can range from work flow and efficiency, time and cost savings to clinical outcomes. In this project, the use of Electronic Health Records in health care management and its impact on patient outcomes were reviewed with a view to assess their impact clinical outcomes only. According to the Working Group on Cardiovascular Disease and Stroke, the term "outcomes" refers to the consequences of treatment and can represent markers of disease progression (mortality, readmission, etc), health status (symptoms, functioning, and quality of life), and/or cost (Brass et al 2000). These measures of success which include death, readmission, physiological end points, patient health status and patient satisfaction have been adopted in the present study. Death, readmission and patient satisfaction are straight forward concepts that probably do not need elaboration here. Thus, throughout this research, careful attention was given to avoid fully embracing the original use of terms, methods, analysis, etc., while at the same time ensuring that all terms, specific results and tables or charts, could be adapted to satisfy the particular context and framework of this study.

\section{FINDINGS}

\section{Overview - Impact of EHRs on Patient Outcomes - 2009 to 2014}

Despite efforts by the federal government to speed up a nation-wide adoption of EHRs, and despite the incentives and the large expenditure to date, less than half of the nation's hospitals have transitioned over to EHRs. As of July 2012, 44 per cent of hospitals in the US had adopted EHR (Charles, King, \& Patel et al, 2013). Although this is triple the rate in 2009, the year HITECH was signed into law, it is quite clear that the government's goal of a nationwide adoption of EHRs is still a long way away from being realized. Besides, at those hospitals or providers that have already implemented EHR it's use is still very much in its infancy, which would further explains the paucity of concrete evidence, on how it is impacting the healthcare.

Thus, an exhaustive and extensive search of the literature, for evidence of the impact of EHRs on the overall healthcare system post-2009, the year of the signing of the HITECH Act, turns up mainly anecdotal evidence, more than expounding the benefits, that could be achieved. Little was said about those benefits that were already been achieved. In addition, there is limited published research findings, regarding the impact of EHRs, on the management of patient health outcomes. This situation underlines the need for more research in this area.

\section{Impact on Clinical Outcomes}

Clinical outcomes generally refer to the presence or absence of disease, symptoms, signs or other abnormality. If the quality of our healthcare is to be improved significantly, managers of healthcare systems must aim at improving clinical patient outcomes such as preventing death, symptoms, or signs. The prevailing view from published research is that EHRs are contributing towards improving patient outcomes and raising the quality of care for a variety of health conditions. Miller \& Tucker (2011) in a 12-county panel study found that hospitals that used EHRs experienced a reduction in neonatal mortality of 16 deaths per 100,000 births. They concluded that this reduction in neonatal mortality particularly the mortality from health conditions that required careful and continuous monitoring was a direct result of the use of EHRs. Similarly, a study by Hung (2011) showed that risk-adjusted mortality rates for patients, with acute myocardial infarction or congestive heart failure, dropped significantly by as much as 0.59 per cent, for hospitals that had implemented and used EHRs (see Table 1). The study identified CPOE as the most significant EHR subsystem responsible for the reduction in risk-adjusted cardiac mortality rate although most of the other subsystems also had significant impact.

The same study also recorded higher magnitudes of risk-adjusted mortality decline rates in hospitals that used EMRs across all units compared with those that used them only in some units consequently he strongly recommended the adoption of EHR systems. A decrease in mortality rate was also reported by Longhurst, Sandborg, Parast et al 
(2010) in their investigation at the Lucile Packard Children's Hospital where over an 18 month period they recorded an astounding 20 percent decrease in mortality rate that coincided with the use of a community CPOE system sold as a subsystem of an HER.

\section{Impact on Patient Perception and Satisfaction}

Patient perception is one of the indices frequently used to assess the quality of healthcare. In fact provider perception of a service or health agency is only as good as the degree of approval it receives from the patients that use it. Perception is dynamic and can be influenced by among other things, past experience, public opinion, education, individual goals, expectations, and fears. Perceptions influence a patient's satisfaction with the care they receive.

On the other hand, patient satisfaction is important to the provider in terms of volume of service provided, financial benefits, and reputation. Most importantly, patient satisfaction is one of the important factors that can affect the patient's level of engagement and participation in the care they are receiving. This is critical since patient satisfaction has been shown to affect patient compliance, treatment outcomes, and the ability to remember instructions (Stewart, Kroth, Schuyler et al, 2010). Patient-centered healthcare is one of the main objectives of the current national health policy. A patient-centered health is also one in which the patient is engaged and actively participates in the care services and disease management plans. The overwhelming evidence in the literature supports the view that EHRs are perceived favorably by most patients.

In a study designed to assess the impact of EHR on diabetes care and outcomes, Herrin, da Graca, Nicewandet et al (2012) observed that by a difference of 9.2 per cent, patients exposed to EHRs were significantly more likely to receive an optimal care bundle than those patients never exposed to EHRs. The optimal care included systolic blood pressure less than $130 \mathrm{~mm} \mathrm{Hg}$, diastolic blood pressure less than $80 \mathrm{~mm} \mathrm{Hg}$. According to Wise (2013), patient satisfaction with both nurses and physicians increased from 50 to 80 per cent when EHR was implemented. Similarly, an impact study by the Sage Healthcare Division, a unit of Sage North America, showed that 81 per cent of patients viewed EHRs very favorably, 45 per cent were very comfortable and not concerned about their physician or clinician using a computer or other electronic device to document their health conditions. Seventy-eight per cent of the patients believed that EHR helped improve the quality of healthcare. Ventres, Kooinga, Vukovic et al (2006) investigating the effects of EHR on physician - patient encounter found patients to be supportive of the view that an EHR session is an appropriate and "to-the-point" encounter.

\section{Impact on Hospital Readmissions}

Among the factors deemed to be responsible for such high rates of hospital readmission are: (1) inadequate relay of information to patients, (2) care givers and post-acute care providers by the planners of the discharging hospital, (3) patient non-compliance with care the instructions issued to them by the discharging physician or nurse, (4) and inadequate follow-up care by post-acute and long-term care providers (Stone \& Hoffman, 2010). Therefore, it seems quite clear that there is a great and urgent need for a patient tracking system such as EHR that will ensure the availability of effective discharge and post-discharge, support services. Metzger, (2012) of The Global Institute for Emerging Healthcare Practices considers that this can only be possible with the help of healthcare information technology. According to her, "A seamless transition to the next provider of care, requires that the physician practice or institution (such as rehab, hospital or skilled nursing facility) be ready to receive the patient and that there will be a common understanding of each patient's status, needs and treatment plan.

Standardizing the format and content of information in the communication is one way of ensuring that all relevant information is included. Presumably, communication will increasingly occur electronically, as both senders and recipients implement electronic health records (EHRs), more advanced features including health information exchange. Tracking patients is essential to ensure planned post-discharge care and support actually occurs in time to address gaps. This will only be possible with the assistance of health IT to accomplish communication and close loops.

Finally, meaningful use for HITECH will provide the critical foundation of much more complete patient information available in the EHR and the basic infrastructure for communicating with clinical partners through health 
information exchange. Hospitals in health systems with integrated EHRs that cross inpatient and physician practice settings have a head start in creating the health IT needed to provide continuity of information during the transition from discharge"(Metzger 2012).

\section{Impact on Patient Monitoring and Compliance}

Simply stated, compliance describes the degree to which a patient correctly follows medical advice or doctor's treatment. Compliance may involve medication, medical devices, prophylactic measures, therapy sessions, physical exercise etc. Both the provider and the patient are important players in ensuring effective compliance. On the other hand, noncompliance can be a major obstacle in achieving quality care for patients. It has been associated with adverse outcomes and higher cost of care. According to Ho, Bryson, \& Rumsfeld (2009) "the achievement of longer-term therapeutic outcome goals requires a partnership with patients." There are several reasons - intentional and non-intentional - for noncompliance. Intentional noncompliance is when the patient weighs the risk and benefits of treatment against any adverse effects and decides against continuing with the course of treatment or medication. Unintentional noncompliance arises from patient's carelessness or forgetfulness to do what is required of them. Whatever the reason, it is clear that there is an urgent need to reduce current rates of noncompliance in order to improve quality of care.

Wise (2013), posited that by implementing electronic health record keeping at Mount Sinai Medical Center in New York, the center realized a range of positive outcomes. First there was an improvement in continuity of care, better communication among care givers, and patient access to prescriptions, appointment and test results. Second, the hospital noticed an increase to almost 100 per cent of its inpatient vaccinations. Thirdly, its real time surveillance reporting resulted in immediate and positive effects on compliance. Lastly, 98 per cent of discharge summaries were available within 30 days post-discharge. Guite, Land, McCartan et al (2013) observed that EHR simplified the work of nursing and led to more appropriate referrals to ancillary departments. According to them, using EHR meant that more than 25 per cent of a patient's information could be imported from a previous encounter thereby eliminating duplicate documentation. In addition, admissions information was being used to monitor and support quality measures. Shaha (2012) reported that vaccination compliance at Robert Wood Johnson University Hospital, New Jersey, achieved significant improvements in safety and quality through the appropriate implementation and use of EHR integrated with advanced Clinical Decision Support (CDS). The hospital used EHR and CDS to automate screening for pneumonia and influenza vaccinations upon discharge. As a result, the hospital experienced an average of 35 per cent improvement in compliance approaching 97.8 per cent. With the EHR CDS system in place the hospital was able to document 100 per cent of all medications and achieve a 90 per cent compliance with home medications per discharge instructions. As a result, many preventable medication errors were avoided.

\section{Impact on Patient Safety}

Patient safety is one area in which there seems to be conflicting evidence as to the benefit of EHRs. There is no doubt that EHRs will require extensive and continuous improvements to make them work better and more efficiently. Of great importance also is the training of personnel in the proper and effective use of EHR systems. If personnel are not trained or only poorly trained, they will be incompetent and prone to many sometimes costly errors. Thus, ironically, while EHR is being promoted as the key to improving patient safety (IOM, 2006; Mitchell, 2008; Staggers, Weir \& Phansalkar, 2008), and even as Bell and Thornton (2011) report that 63 per cent fewer errors and a 60 per cent decrease in near-miss medication events among hospitals using EHRs, some studies have however reported serious problems associated with the use of EHR. One such study is that by Magrabi, Ong, Runcinamet al (2011) who investigated health information technology events associated with patient harm that were submitted to the US Food and Drug Administration (FDA) Manufacturer and User Facility Device Experience (MAUDE) database. They found that CPOE and Picture Archiving and Communication Systems (PACS) were involved in 93 per cent of the reported events. Forty-one per cent of the adverse events were associated with medication, 33 per cent with clinical processes, 15 per cent with radiation, and 11 per cent with surgery. There were four deaths. The authors recommend that strategies to improve safety of Health Information Technology should focus on designing safer user interfaces, integrated checks of key identifiers and decision support, and engineering of safer clinical processes. According to Sparnon \& Marella (2012) "The majority of EHR-related reports involved 
errors in human data entry, such as entry of "wrong" data or the failure to enter data, and a few reports indicated technical failures on the part of the EHR system."

\section{CONCLUSIONS}

The use of Electronic Health Records in the US has soared within the past few years with more hospitals and officebased physicians implementing at least some basic form of the technology. However, the nation is still a long way away from realizing its stated goal of having every healthcare provider use EHRs. There is a dearth of published empirical data on the impact of EHRs post-2009 the year of the HITECH Act possibly because the national adoption program is still very much in its early stages. But from the little that is available, it is quite evident that EHRs are already having a positive impact on the quality of healthcare in several areas of patient outcomes - clinical outcomes, perception and satisfaction, monitoring, compliance, readmissions, and safety. Based on the positive outcomes reported in these areas, it can be concluded that EHRs hold great potential and promises significant improvement in our healthcare. Indeed, EHRs are the face of our future healthcare delivery. Therefore, it is high time that our healthcare system caught up with the tremendous advances in information technology and changes in our society.

\section{FUTURE RESEARCH}

A key area that this paper did not address is the framework of cyber security. An emerging model for cyber-security is the notion that cyber-security may be standardized via four continuous functions: Identify, Protect, Detect, Respond, and Recover. The framework core, in effect, describes the continuous cycle of business processes that constitute effective cyber security.

\section{REFERENCES}

AHRQ PSNet Patient Safety Network. Patient safety. [Accessed October 20, 2013]. http://psnet .ahrq.gov/glossary.aspx\#P

Alvarez, R. (2005). Health Care Has to Move into the Hi-Tech Age. Bull World Health Organ, 83(5): 323-323.

American Heart Association/American College of Cardiology (AHA/ACC). First Scientific Forum on Assessment of Healthcare Quality in Cardiovascular Disease and Stroke. Quality of Care and Outcomes Research in CVD and Stroke Working Groups1. Circulation. 2000;101:1483-1493.

Ashish, J.H.A. (2013). As the Debate Over Obama care Implementation Rages, a Success on the IT Front. http://thehealthcareblog.com/blog/2013/07/12/as-the-debate-over-obamacare-implementation-rages-asuccess-on-the-it-front/

Bates, D.W. \& Gawande, A. (2003. Improving Safety with Information Technology. New England Journal of Medicine, 348: 2526-2534.

Bell, B., Thornton, K. (2011). From promise to reality achieving the value of an EHR. Healthcare Financial Management, 65(2), 51-56.

Center for Medicare and Medicaid Services (CMS) (2013). Medicare and Medicaid EHR Incentive Program Basics. https://www.cms.gov/Regulations-and-uidance/Legislation/EHRIncentivePrograms/Basics.html 
Centers for Medicare \& Medicaid Services (CMS). Medical Hospital Quality Chartbook 2011, Performance Report on Readmission Measures for Acute Myocardial Infarction, Heart Failure, and Pneumonia, September 15, 2011.https://www.cms.gov/Medicare/Quality-Initiatives-Patient-AssessmentInstruments/HospitalQualityInits/Downloads/HospitalChartBook2011.pdf

Chan, K., Fowles, J., Weiner, J. (2010). Electronic health records and the reliability and validity of quality measures: a review of the literature. Med Care Res Rev, 67: 503e27.

Charles, D., King, J., Patel, V., Furukawa, M.F. (2013).Adoption of Electronic Health Record Systems among U.S. Non-federal Acute Care Hospitals: 2008-2012 . ONC Data Brief No. 9. March 2013.

Congressional Research Service. "Medicare Hospital Readmissions: Issues, Policy Options and PPACA, 2010.’http://www.hospitalmedicine.org/AM/pdf/advocacy/CRS_Readmissions_Report.pdf

Galanter, W.L., Hier, D.B., Jao, C. et al (2010). Computerized physician order entry of medications and clinical decision support can improve problem list documentation compliance. Int J Med Inform, 79: 332e8.

Gonzalez, J. (2013). The Case for Electronic Health Records. SecureEDI http://www.secureedi.com/documents/the_case_for_electronic_health_record.pdf Accessed, October 7, 2013

Guite, J., Lang, M., McCartam, P., \& Miller, J. (2013). Nursing Admissions Process Redesigned to Leverage EHR. Journal of Healthcare Information Management, 20: No. 2.

Health Information and Management Systems Society (HIMSS). EHR: electronic health record. http://www.himss.org/ASP/topics_ehr.asp. Accessed October 15, 2013.

Healthcare Information and Management Systems Society (HIMSS). Electronic health record attributes and essential requirements (2003). Available at: http://www.himss.org/content/files/ehrattributes0707 03.pdf. Accessed October 22, 2013.

Hopkins, R. (2013). Why is EHR Use Inconsistent Among Doctors? Published on McCombs TODAY June 17,2013 - 1:52pm. http://www.today.mccombs.utexas.edu

Hung, P. (2011). Effects of Electronic Health Record Adoption on Hospital Risk-Adjusted Mortality Rates Patients with Acute Myocardial Infarction or Congestive Heart Failure. A thesis submitted to the Faculty of the Rollins School of Public Health of Emory University, Atlanta, Georgia in partial fulfillment of the requirements for the degree of Master of Sciences in Public Health in Health Policy and Health Services Research. 2011.

Institute of Medicine (IOM). Crossing the Quality Chasm: A New Health System for the 21st Century. Washington, D.C. National Academy Press, 2001.

Institute of Medicine (IOM). Preventing medication errors: Quality chasm series. Washington, DC: National Academies Press; 2006.

Institute of Medicine (IOM). To err is human: Building a safer health system. Washington, DC: National Academy Press; 2000.

Institute of Medicine (IOM): Committee on the Quality of Health Care in America. Crossing the quality chasm: A new health system for the 21st century. Washington, DC: National Academy Press; 2001.

Longhurst, C.A., Parast, L., Sandborg, C.I. et al (2010). Decrease in Hospital-wide Mortality Rate after Implementation of a Commercially Sold Computerized Physician Order Entry System. Pediatrics ,126;14 
Magrabi, F., Ong, M., Runciman, W., \& Coiera, E. (2011). Patient Safety Problems Associated with Heathcare Information Technology: an Analysis of Adverse Events Reported to the US Food and Drug Administration. AMIA AnnuSymp Proc. 2011; 2011: 853-857. Published online 2011 October 22.

Metzger, J. (2012). Preventing Hospital Readmissions: The First Test Case for Continuity of Care. Global Institute for Emerging Healthcare Practices: a division of Computer Services Corporation (CSC).

Miller, A.R. \& Tucker, C.E. (2011). Can Healthcare Information Technology Save Babies? Journal of Political Economy, Vol. 119, No. 2, April 2011. The University of Chicago Press

Mitchell, P.H. (2008). In "Patient Safety and Quality: An Evidence-Based Handbook for Nurses." U.S. Department of Health and Human Services, 540 Gaither Road Rockville, MD 20850 Editor: Ronda G. Hughes. $A H R Q$ Publication No. 08-0043. April 2008.

Parsons, A., McCullough, C., Wang, J., \& Shih, S. (2012). Validity of electronic health record-derived quality measurement for performance monitoring. J Am Med Inform Assoc, 19:604-609.

Poissant, L., Pereira, J., Tamblyn, R. (2005). The Impact of Electronic Health Records on Time Efficiency of Physicians and Nurses: A Systematic Review. Journal of the American Medical Informatics Association, 12(5): 505-516.

Reed, M., Huang, J., Brand, R., Graetz, I., et al (2013). Implementation of an Outpatient Electronic Health Record and Emergency Department Visits, Hospitalizations, and Office Visits among Patients with Diabetes. JAMA, 310(10): 1060-1065.

Shaha, S. (2012). All EHRs Are Not Created Equal: Improving Outcomes with Advanced CDS. http://blog.allscripts.com/2012/02/23/all-ehrs-are-not-created-equal-improving-outcomes-with-advancedcds/

Sparnon, E., Marella, W. M. (2012).The Role of the Electronic Health Record in Patient Safety Events. Pa Patient SafAdvis, 2012 Dec; 9(4):113-21.

Staggers, N., Charlene, Weir., \& Phansalkar, S. Chapter 47. Patient Safety and Health Information Technology: Role of the Electronic Health Record.

Staggers, N., Weir, C., \& Phansalkar, S. (2008). Chapter 47. Patient Safety and Health Information Technology: Role of the Electronic Health Record. In "Patient Safety and Quality: An Evidence-Based Handbook for Nurses”. Editor: Ronda G. Hughes. AHRQ Publication No. 08-0043 April 2008.

Stewart, R.F., Kroth, P.J., Schuyler, M., Bailey, R. (2010). Do electronic health records affect the patientpsychiatrist relationship? A before \& after study of psychiatric outpatients. BMC Psychiatry, 10(3):1-9.

Stone, J., Hoffman, G.J. (2010). Congressional Report Service September 21, 2010. Congressional Research Service. 7-5700. www.crs.gov R40972

Versel, N. (2013). Topics in Health: Lessons From The Field. Covering Electronic Health Records. Know Your Context and Lingo, from HIT to PHRs to EMRs http://www.reportingonhealth.org/resources/lessons/covering-electronic-health-records. Accessed October $18,2013$.

Wise, .P (2013). THE EHR: Providing Exceptional Value. http://www.himss.org/ResourceLibrary/ResourceDetail.aspx?ItemNumber=17246. February 26, 2013

Wolfe, T.E. (2008). Making the Case for Electronic Health Records: A Report from ASCO's EHR Symposium. $J$ Oncol Pract, 4(1): 41-42. 